\title{
Loss of Tdp-43 disrupts the axonal transcriptome of motoneurons accompanied by impaired axonal translation and mitochondria function
}

Michael Briese ${ }^{1 \dagger}$, Lena Saal-Bauernschubert ${ }^{1 \dagger}$, Patrick Lüningschrör ${ }^{1 \dagger}$, Mehri Moradi $^{1 \dagger}$, Benjamin Dombert ${ }^{1}$, Verena Surrey ${ }^{1}$, Silke Appenzeller ${ }^{2}$, Chunchu Deng ${ }^{1}$, Sibylle Jablonka ${ }^{1}$ and Michael Sendtner ${ }^{1 *}$ (D)

\begin{abstract}
Protein inclusions containing the RNA-binding protein TDP-43 are a pathological hallmark of amyotrophic lateral sclerosis and other neurodegenerative disorders. The loss of TDP-43 function that is associated with these inclusions affects post-transcriptional processing of RNAs in multiple ways including pre-mRNA splicing, nucleocytoplasmic transport, modulation of mRNA stability and translation. In contrast, less is known about the role of TDP-43 in axonal RNA metabolism in motoneurons. Here we show that depletion of Tdp-43 in primary motoneurons affects axon growth. This defect is accompanied by subcellular transcriptome alterations in the axonal and somatodendritic compartment. The axonal localization of transcripts encoding components of the cytoskeleton, the translational machinery and transcripts involved in mitochondrial energy metabolism were particularly affected by loss of Tdp43. Accordingly, we observed reduced protein synthesis and disturbed mitochondrial functions in axons of Tdp-43depleted motoneurons. Treatment with nicotinamide rescued the axon growth defect associated with loss of Tdp43. These results show that Tdp-43 depletion in motoneurons affects several pathways integral to axon health indicating that loss of TDP-43 function could thus make a major contribution to axonal pathomechanisms in ALS.
\end{abstract}

Keywords: Amyotrophic lateral sclerosis, Tdp-43, Axonal transcriptome, Nicotinamide

\section{Introduction}

In neurons, mechanisms for subcellular mRNA transport and translation in dendrites and axons allow the regulation of localized responses to cellular stress conditions. Alterations in these RNA processing mechanisms can lead to neural dysfunction and neurodegeneration [1-3]. Likewise, disturbed subcellular RNA distribution has been found to be

* Correspondence: Sendtner_M@ukw.de

${ }^{\dagger}$ Michael Briese and Lena Saal-Bauernschubert are co-first authors.

${ }^{\dagger}$ Patrick Lüningschrör and Mehri Moradi contributed equally to this work.

${ }^{1}$ Institute of Clinical Neurobiology, University of Wuerzburg, 97078 Wuerzburg, Germany

Full list of author information is available at the end of the article a pathophysiological hallmark in amyotrophic lateral sclerosis (ALS) [4-6] and spinal muscular atrophy (SMA) [7].

Subcellular mRNA transport is mediated by messenger ribonucleoprotein particles (mRNPs) which contain RNA-binding proteins as essential components. One of these RNA-binding proteins is the transactive response DNA-binding protein (TARDBP or TDP-43) [4]. Initially, TDP-43 was identified as a strong transcriptional repressor of human immunodeficiency virus gene expression by binding to the TAR DNA in transfected cells [8]. Since then, multiple roles in RNA processing have been identified for TDP-43 such as regulation of premRNA splicing, translation, miRNA processing, mRNA stability and subcellular transport [9-11]. Under 
physiological conditions, TDP-43 is predominantly found in the nucleus although the protein is able to shuttle between the nucleus and the cytoplasm [12], suggestive of a role in mRNA stabilization and mRNP trafficking from the nucleus to the cytoplasm, in addition to its nuclear function in transcriptional regulation.

ALS pathology is generally characterized by degeneration of upper and lower motoneurons, resulting in a dramatic loss of motor function [13]. Disruption of axonal RNA transport might contribute to the specific pathophysiology in motoneurons [14]. For example, the motility of axonal TDP-43 transport granules is impaired when mutant TDP-43 harboring ALS-associated mutations is expressed in neurons [4]. In line with this finding, the anterograde axonal transport of TDP-43associated mRNA granules is affected in induced pluripotent stem (iPS) cell-derived motoneurons from ALS patients [4]. Axonal transport defects have also been reported in Drosophila models of ALS [15], suggesting a conserved cellular function of TDP-43 in subcellular localization of RNA. RNA immunoprecipitation sequencing (RIP-seq) and crosslinking and immunoprecipitation (CLIP) studies revealed specific binding sites of TDP-43 along single transcripts and identified transcript level changes of several mRNAs after TDP-43 depletion [16-18]. Preferential binding of TDP-43 to the 3'UTRs of specific mRNAs was found for cytoplasmically localized TDP-43, which might be reflective of its role in subcellular mRNA localization [18].

In order to investigate how TDP-43 is involved in modulation of axonal functions, we determined the consequences of Tdp- 43 depletion in primary mouse motoneurons both on the morphological level and by analysis of alterations in the somatodendritic and axonal transcriptomes. Tdp- 43 knockdown significantly impaired axon elongation. RNA-seq analysis revealed that many transcripts relevant for axonal cytoskeletal integrity, mitochondrial function and translation were dysregulated in the axonal compartment upon Tdp-43 depletion. In agreement with this finding, protein translation and mitochondrial functionality were specifically impaired in axons of Tdp-43-deficient motoneurons. Finally, we found that treatment with nicotinamide, a precursor for the cofactor $\mathrm{NAD}+$ that is required for oxidative phosphorylation, restored axon growth in Tdp-43 knockdown motoneurons to normal levels. Our results therefore suggest that reduction of Tdp-43 function dysregulates RNA transport processes that are vital for axon growth, local protein synthesis and energy production.

\section{Materials and methods}

\section{Animals}

CD-1 mice were housed in the animal facility of the Institute of Clinical Neurobiology at the University
Hospital of Wuerzburg. Mice were maintained at a $12 \mathrm{~h} /$ $12 \mathrm{~h}$ day/night cycle under controlled conditions in 55$65 \%$ humidity. Food and water were available in abundant supply. Each experiment was conducted following the regulations on animal protection of the German federal law and of the Association for Assessment and Accreditation of Laboratory Animal Care, in agreement with and under control of the local veterinary authority.

\section{Isolation and culture of primary embryonic mouse motoneurons}

Spinal cords were dissected from E12.5 CD-1 mouse embryos as described previously [19]. Dorsal root ganglia and meninges were removed and tissue was digested by $0.1 \%$ trypsin (Worthington). Motoneurons were enriched by incubation with anti-p75 antibody-coated (MLR2, Abcam) cell culture dishes. For axon length analysis, 2000 cells were plated per glass coverslip and cultured in the presence of BDNF $(5 \mathrm{ng} / \mathrm{ml})$ in neurobasal medium (Invitrogen) containing $500 \mu \mathrm{M}$ GlutaMAX (Invitrogen), $2 \%$ horse serum (Gibco) as well as $2 \%$ B27 supplement (Invitrogen) for $7 \mathrm{~d}$ at $37^{\circ} \mathrm{C}$ with $5 \% \mathrm{CO}_{2} .50 \%$ of the culture medium was replaced at $1 \mathrm{~d}$ in vitro (DIV1) and then every second day. For nicotinamide treatment, medium was supplemented with nicotinamide (Applichem) at the indicated concentrations. Motoneuron cultures in compartmentalized chambers were performed as previously described [7]. CNTF was used at a concentration of $5 \mathrm{ng} / \mathrm{ml}$ on the somatodendritic and axonal side and BDNF at a concentration of $20 \mathrm{ng} / \mathrm{ml}$ on the axonal side.

\section{Knockdown of Tdp-43 via lentiviral shRNA in embryonic mouse motoneurons}

Four different siRNA sequences targeting $T d p-43$ were designed using the siRNA Wizard (Invivogen). The oligonucleotides for cloning the corresponding shRNAs into the pSIH-H1 shRNA vector (System Bioscience) are listed in Supplementary Table 1. All vectors generated in this study were sequenced in order to verify their integrity. HEK293T cells were used for virus production as described previously [20]. For lentiviral transduction, motoneurons were incubated for $10 \mathrm{~min}$ at room temperature (RT) with knockdown or control virus prior to plating. $T d p-43$ transcript levels were measured by qPCR using Gapdh as housekeeping transcript.

\section{Immunofluorescence staining of free-floating spinal cord sections}

Free-floating sections were essentially prepared as described before [21]. Mice were transcardially perfused with 4\% paraformaldehyde (PFA). Subsequently, the spinal cord was removed and post-fixed in 4\% PFA for 2 $\mathrm{h}$ at RT. After embedding in 5\% agarose, $40 \mu \mathrm{m}$ thick, 
free-floating sections were cut on a Leica VT1000 S microtome and collected in $0.1 \mathrm{M}$ phosphate buffer $\mathrm{pH}$ 7.4. After incubation for $2 \mathrm{~h}$ at RT with blocking solution [ $10 \%$ donkey serum, $0.3 \%$ Triton $\mathrm{X}-100$ and $0.1 \%$ Tween-20 in Tris-buffered saline (TBS)], the sections were incubated for $72 \mathrm{~h}$ at $4{ }^{\circ} \mathrm{C}$ with primary antibodies in 1:10 diluted blocking solution (1\% donkey serum, $0.03 \%$ Triton X-100 and $0.01 \%$ Tween-20 in TBS). After washing three times for $10 \mathrm{~min}$ in TBS-Tween (TBS-T) at RT, sections were incubated for $2 \mathrm{~h}$ at RT with the appropriately fluorophore-conjugated secondary antibodies, washed again in TBS-T and finally mounted with Fluor Save (Merck-Millipore). The following primary antibodies were used: polyclonal goat anti-ChAT (MAB144P, Millipore; 1:1000) and polyclonal rabbit anti-TDP43 (10782-2-AP, Proteintech; 1:1000).

\section{Immunofluorescence staining of cultured motoneurons}

Motoneurons were washed with phosphate-buffered saline (PBS) and fixed with 4\% PFA for $15 \mathrm{~min}$ at RT. For permeabilization, $0.3 \%$ Triton X-100 was applied for 20 min at RT. Motoneurons were treated with $2 \%$ donkey serum, 2\% BSA, 2\% saponin and 5\% sucrose in PBS for at least $1 \mathrm{~h}$ to reduce unspecific binding followed by primary antibody incubation overnight at $4{ }^{\circ} \mathrm{C}$. Motoneurons were washed thrice and incubated with secondary antibodies for $1 \mathrm{~h}$ at RT. Nuclei were visualized by DAPI and motoneurons were embedded with Aqua Poly/ Mount (18606, Polysciences). The following primary and secondary antibodies were used for immunostaining: polyclonal rabbit anti-TDP-43 (10782-2-AP, Proteintech; 1:300), monoclonal mouse anti- $\alpha$-Tubulin (T5168, Sigma-Aldrich; 1:1000), donkey anti-rabbit (H+L) IgG (Cy3; 711-165-152, Jackson Immunoresearch; 1:500) and goat anti-mouse ( $\mathrm{H}+\mathrm{L})$ IgG (Cy5; 115-175-146, Jackson Immunoresearch; 1:500).

\section{Axon length measurements}

Motoneurons transduced with lentiviruses were immunostained at DIV7 with rabbit polyclonal anti-Tau (T6402, Sigma-Aldrich; 1:800) and chicken polyclonal anti-GFP (ab13970, Abcam; 1:2000) antibodies. The length of the longest axon branch was measured using ImageJ software; axon collaterals were not considered for the analysis. Motoneurons were only scored when designated axons were at least three times longer than the corresponding dendrites ensuring an unambiguous distinction between axons and dendrites. Statistical analysis was performed using GraphPad Prism software (GraphPad Software).

\section{RNA-seq analysis}

Whole transcriptome amplification and high-throughput sequencing of RNA from compartmentalized motoneurons were performed as previously described [22]. Briefly, total RNA was extracted from the somatodendritic and the axonal compartment of compartmentalized motoneurons and reverse-transcribed. Following second strand synthesis, fragments were PCR amplified and converted into highthroughput sequencing libraries. Sequencing, read mapping and data analysis were performed as described before [22]. Control datasets from motoneurons transduced with empty lentiviral expression vector were described before [1]. Control and Tdp-43 knockdown motoneurons were processed in parallel for RNA-seq. The sequencing data described in this publication are accessible in NCBI's Gene Expression Omnibus [23] through GEO Series accession number GSE147607. For comparison with TDP-43 iCLIP, we used iCLIP data from control subjects from Tollervey et al. [18]. For GO term analysis we used the Database for Annotation, Visualization and Integrated Discovery (DAVID) [24]. As background datasets, we used the 10,433 and 11,127 transcripts detected in the somatodendritic and axonal compartment, respectively, of wildtype motoneurons [22].

\section{Puromycin labeling}

After 6 DIV, primary motoneurons were labeled with puromycin $(10 \mu \mathrm{g} / \mathrm{ml})$ for $15 \mathrm{~min}$. After $15 \mathrm{~min}$, the cells were washed once with Hanks' Balanced Salt Solution (HBSS) (Gibco), and fresh medium was added. The puromycin incorporation was chased for $45 \mathrm{~min}$. For depolymerization of microtubules, cells were treated with $10 \mu \mathrm{M}$ nocodazole for $2 \mathrm{~h}$ prior to puromycin labeling. For inhibition of protein synthesis, cultured motoneurons were treated with cycloheximide $(10 \mu \mathrm{g} / \mathrm{ml})$ or anisomycin $(100 \mathrm{ng} / \mathrm{ml})$ for $1 \mathrm{~h}$ prior to puromycin labeling. Subsequently, the cells were fixed with $2 \%$ PFA for $15 \mathrm{~min}$ at RT. Puromycin incorporation was visualized with an anti-puromycin antibody (clone 12D10, MABE343, Merck Millipore; 1:1000). Motoneurons were counterstained with an anti-Tau antibody (T6402, Sigma-Aldrich; 1:500).

\section{Measuring mitochondrial membrane potential in live mouse motoneurons}

Mitochondria were labeled with MitoTracker ${ }^{\mathrm{TM}}$ Orange CM-H2TMRos (M7511, Thermo Fisher Scientific) and TMRM (Tetramethylrhodamine, Methyl Ester, Perchlorate, T668, Thermo Fisher Scientific) was used to determine the mitochondrial membrane potential $(\Delta \psi \mathrm{m})$. Motoneurons were cultured on laminin-111-coated (23017-015, Invitrogen) $35 \mathrm{~mm}$ Ibidi $\mu$-dishes $(81,156$, Ibidi) for $6 \mathrm{~d}$. Cells were washed twice with pre-warmed Tyrode's buffer [125 mM NaCl, $2 \mathrm{mM} \mathrm{KCl,} 30 \mathrm{mM}$ glucose, $2 \mathrm{mM} \mathrm{CaCl}_{2}, 2 \mathrm{mM} \mathrm{MgCl}_{2}$, and $25 \mathrm{mM} \mathrm{HEPES}$ (pH 7.4)] and $20 \mathrm{nM}$ TMRM or MitoTracker ${ }^{\mathrm{TM}}$ Orange were loaded into cells by incubation for $15 \mathrm{~min}$ at $37^{\circ} \mathrm{C}$ and $5 \% \mathrm{CO}_{2}$. Then, motoneurons were washed twice 
with pre-warmed Tyrode's buffer and imaged in $2 \mathrm{ml}$ Tyrode's buffer supplemented with $5 \mathrm{ng} / \mathrm{ml}$ BDNF. Livecell imaging was conducted on an inverted epifluorescence microscope (TE2000, Nikon) equipped with 60x 1.4-NA objective, Perfect Focus System (Nikon), a Cool LED pE-4000 light source and an Orca Flash 4.0 V2 camera (Hamamatsu Photonics). During live-cell imaging, cells were maintained at $37^{\circ} \mathrm{C}$ and $5 \% \mathrm{CO}_{2}$ on a heated stage chamber (TOKAI HIT CO., LTD). TMRM and MitoTracker ${ }^{\text {rm }}$ Orange CM-H2TMRos were excited at $560 \mathrm{~nm}$ illumination using $2 \%$ light intensity and 12bit images of $1024 \times 1024$ pixels were acquired for $5 \mathrm{~min}$ at $30 \mathrm{~s}$ intervals. Mitochondria labeled with MitoTracker $^{\text {Tx }}$ Orange were counted in axons of control and Tdp-43 knockdown motoneurons and normalized to axon length. ImageJ was used to measure the mean grey value of TMRM fluorescence intensity per mitochondrion and average background intensity was subtracted. Median intensity values of mitochondria per axon were normalized to the average intensity of the corresponding control group. In control experiments with FCCP, mitochondria labeled with TMRM were imaged first for $1 \mathrm{~min}$ with $30 \mathrm{~s}$ intervals. Next, $10 \mu \mathrm{M}$ FCCP (C2920, Merck) was added and axons were imaged for another $8 \mathrm{~min}$. FCCP depolarizes the mitochondrial membrane potential, resulting in a rapid and significant decrease in the fluorescent intensity of TMRM upon 1 min addition.

\section{qPCR}

RNA was reverse-transcribed with random hexamers using the First Strand cDNA Synthesis Kit (Thermo Fisher Scientific). Reverse transcription reactions were diluted 1:5 in water. Reactions were set up with Luminaris HiGreen qPCR Master Mix (Thermo Fisher Scientific) on a LightCycler 96 thermal cycler (Roche). Primers are listed in Supplementary Table 2.

\section{Results}

Tdp-43 protein localizes to axons and growth cones of primary mouse motoneurons and regulates axon growth In order to characterize the subcellular distribution and in particular the location and function of Tdp- 43 in axons we first investigated the Tdp-43 distribution in spinal cord of adult mice by immunostaining (Fig. 1a). An antibody against acetylcholine transferase (ChAT) was used to identify motoneurons. In all ChAT-positive cells, Tdp-43 was prominently detectable both in the nucleus and the cytoplasm. To confirm this observation, we investigated the distribution of $\mathrm{Tdp}-43$ in primary mouse motoneurons cultured for $6 \mathrm{~d}$ (Fig. 1b). An antibody against $\alpha$-Tubulin was used for staining of axons. Tdp-43 immunoreactivity was detectable in the nucleus and in the cytoplasm, including axons, of motoneurons. Interestingly, Tdp- 43 protein was not distributed in a diffuse manner but localized to distinct axonal structures. As a specificity control, these structures remained unlabelled when Tdp-43 expression was suppressed by shRNA expression (Fig. 1c, see below for details). This staining pattern resembled the axonal Tdp- 43 distribution reported previously [25]. Positive structures in the axon probably include mitochondria that have been identified to bind TDP-43 $[26,27]$ but possibly also particles for axonal RNA transport and processing [4, 25]. Interestingly, such Tdp-43-positive spot-like structures appeared highly enriched in axonal growth cones of primary motoneurons (Fig. 1b).

To elucidate the function of Tdp-43 in motoneurons, we measured axon growth of Tdp-43-depleted embryonic motoneurons that were cultured for $7 \mathrm{~d}$. For this purpose, we designed four different shRNAs (shTdp43.1-4) targeting the $T d p-43$ transcript and cloned them into a lentiviral expression vector. The lentiviral constructs also expressed GFP for visualization of virus infection. We transduced primary motoneurons with these different Tdp-43 knockdown viruses or a control virus expressing a Luciferase shRNA (shLuc) and cultured them for $7 \mathrm{~d}$ (Fig. 2a). At this time point Tdp-43 knockdown lentiviruses expressing sh $T d p-43.1,2$ or 3 reduced $T d p-43$ transcript levels by $57-71 \%$ relative to controls (Fig. 2b). In contrast, Tdp-43 knockdown virus expressing $\operatorname{sh} T d p-43.4$ was not effective in reducing $T d p-43$ mRNA levels. We then investigated the effect of Tdp- 43 knockdown on axon growth. Motoneurons were immunostained for the axonal marker protein Tau and axon length was measured at DIV7 (Fig. 2a, c). Axon lengths of motoneurons transduced with $\operatorname{sh} T d p-43.1,2$ or 3 were significantly reduced relative to controls. In agreement with the qPCR results for measuring knockdown efficacy, transduction with shTdp-43.4 had no significant effect on axon outgrowth. As transduction with shTdp43.2 showed the highest reduction in axon length, we proceeded with this shRNA (denoted 'shTdp-43' hereafter) for all the subsequent Tdp-43 knockdown experiments and used it also for confirmation of the specificity of the Tdp-43 immunolabeling procedure (Fig. 1c).

\section{Loss of Tdp-43 dysregulates the somatodendritic and axonal transcriptome}

To find out whether the disturbed axonal elongation of Tdp-43-deficient motoneurons could be due to subcellular transcriptome alterations we performed RNA-seq on the somatodendritic and axonal compartment of Tdp- 43 knockdown motoneurons grown in microfluidic chambers [7]. For this purpose, we cultured the motoneurons for $7 \mathrm{~d}$ in these specialized culture dishes and then separately isolated total RNA from the two compartments. Due to the low amount of RNA that can be extracted from the axonal compartment we used a previously 


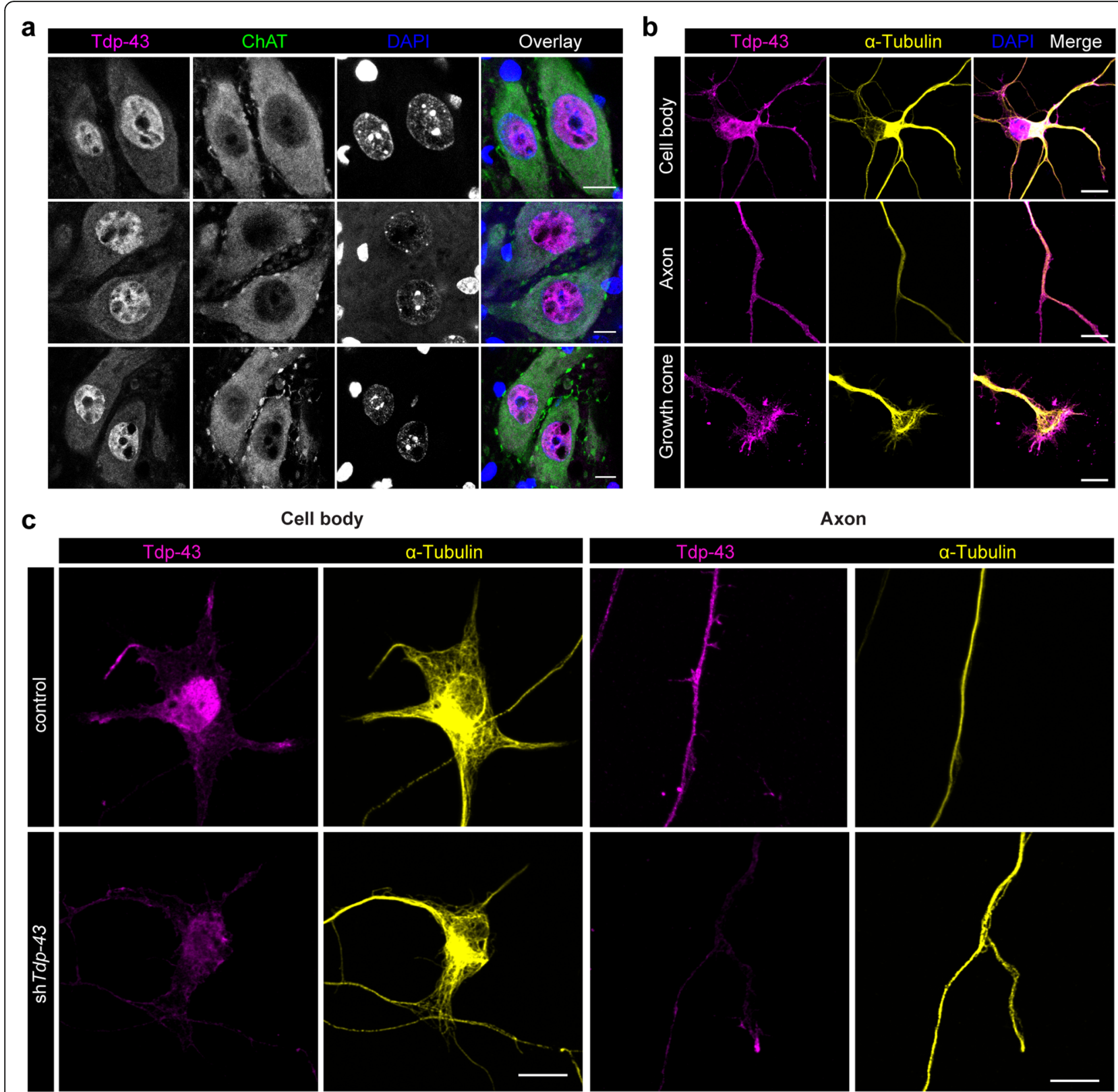

Fig. 1 Tdp-43 localizes in the nucleus and cytosol of motoneurons. a Spinal cord cross sections of adult CD-1 mice immunostained with an antibody against Tdp-43 and an antibody against choline acetyltransferase (ChAT) as a motoneuron marker. DAPI was used for visualization of nuclei. Scale bar: $10 \mu \mathrm{m}$. b Primary mouse motoneurons (DIV6) were immunostained with an antibody against Tdp-43 and with an antibody against a-Tubulin. DAPI was used for visualization of nuclei. Scale bar: $10 \mu \mathrm{m}$. c Immunostaining of motoneurons transduced with empty lentiviral expression vector (control) or vector expressing a shRNA targeting Tdp-43 (shTdp-43) with antibodies against Tdp-43 and Tubulin. Scale bar: $10 \mu \mathrm{m}$

established pre-amplification protocol for RNA-seq in order to obtain sufficient levels of cDNA from each compartment for high-throughput sequencing [22]. Furthermore, this protocol enabled us to analyze whole transcriptome changes without any selection for RNA subtypes, and therefore also included noncoding RNAs and ribosomal RNAs (rRNAs).
We observed an $85 \%$ reduction of $T d p-43$ transcript levels by qPCR in Tdp-43 knockdown motoneurons transduced with $\operatorname{sh} T d p-43$ relative to control motoneurons transduced with an empty lentiviral expression construct (Fig. 3a). Following RNA-seq a similar depletion of Tdp43 transcripts could be measured as FPKM (Fig. 3a). To identify transcripts dysregulated in the somatodendritic 


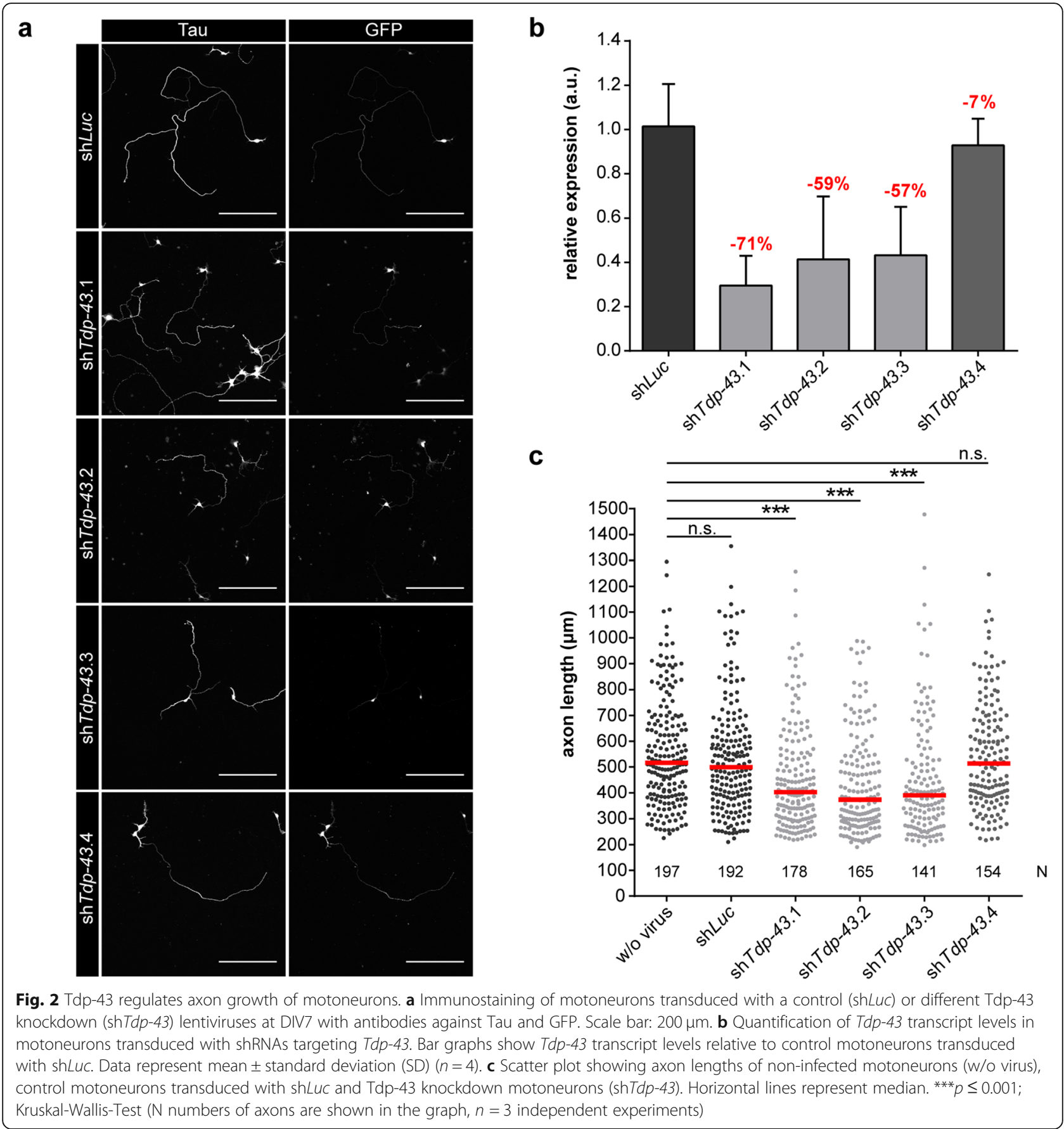

and axonal compartment of Tdp-43 knockdown motoneurons relative to control motoneurons we performed differential expression analysis (Fig. 3b). This revealed 658 transcripts that were significantly $(p<0.05)$ dysregulated in the somatodendritic compartment (Fig. 3c). Of these, 371 were upregulated (Supplementary Table 3) and 287 downregulated (Supplementary Table 4) upon Tdp-43 suppression. The Tdp-43 transcript was the second most strongly decreased transcript which underscores the validity of our RNA-seq analysis. In the axonal compartment of Tdp-43 knockdown motoneurons, 254 transcripts were significantly dysregulated relative to controls (Fig. 3c). Of these, 118 transcripts were upregulated (Supplementary Table 5) and 136 downregulated (Supplementary Table 6). When we compared the dysregulated transcripts in the somatodendritic and axonal compartment, we identified a group of 96 transcripts that were significantly dysregulated in the axonal and somatodendritic compartments upon Tdp-43 loss $(p<0.05$ in both axonal and somatodendritic compartment) (Supplementary Table 7) (Fig. 3c). Of these 


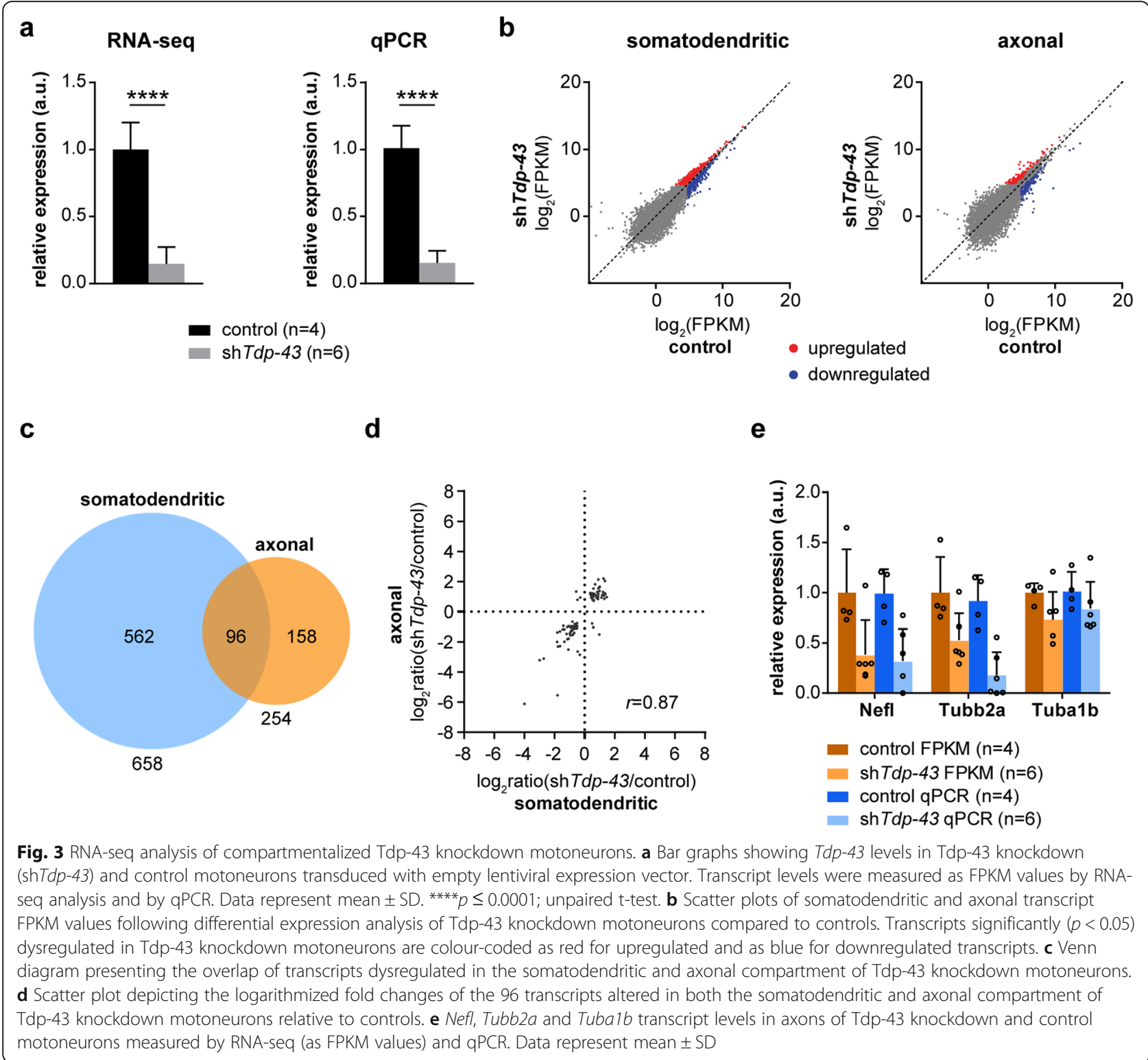

96 transcripts, 47 transcripts were downregulated and 43 transcripts were upregulated in either compartment. The magnitude of change of these transcripts was highly correlated between the two compartments (Pearson's $r=0.87$ ) (Fig. 3d). These transcript alterations that are common to both compartments are likely due to global changes in transcription and/or stabilization, in line with functions of Tdp-43 in transcription and mRNA stability $[9,11]$.

We observed that several transcripts encoding for components of the cytoskeleton were downregulated. Among these were the transcripts encoding Neurofilament light chain polypeptide (Nefl), Tubulin beta-2A (Tubb2a) and Tubulin alpha-1B (Tuba1b). We validated the downregulation of these transcripts in axons of Tdp43 knockdown motoneurons by qPCR (Fig. 3e). Nefl
mRNA binds to TDP-43 and has been shown previously to be transported anterogradely as part of Tdp-43containing granules in axons of mouse cortical neurons $[4,28]$. Beyond that, Nefl mRNA levels are known to be reduced in degenerating motoneurons from ALS patients $[29,30]$. Our result substantiates these findings by showing that loss of Tdp-43 in mouse motoneurons impairs axonal levels not only of Nefl but also of transcripts encoding other cytoskeletal components.

\section{Analysis of Tdp-43 binding on transcripts dysregulated by Tdp-43 deficiency}

Next, we investigated the relationship between Tdp- 43 binding to transcripts and their alterations in Tdp- 43 knockdown motoneurons. For this purpose, we overlaid our RNA-seq 
datasets with the TDP-43 iCLIP data generated from human brain [18]. We found that those transcripts that were upregulated in the axonal compartment of Tdp- 43 knockdown motoneurons contain a higher number of iCLIP hits than downregulated or unchanged transcripts (Fig. 4a). Since axonal transport of mRNAs is usually mediated by interactions of RNA-binding proteins with motifs in the 3'UTRs of these mRNAs [31] we next investigated the ratio of iCLIP hits in the 3'UTR compared to the total number of hits. The cumulative frequency distribution of this ratio showed that transcripts downregulated in the axonal or somatodendritic compartment of Tdp-43 knockdown motoneurons contained a higher proportion of iCLIP hits in their 3'UTRs compared to unchanged or upregulated transcripts (Fig. 4b). This effect is even more pronounced for those axonal transcripts that are selectively altered in the axonal but not somatodendritic compartment of Tdp-43 knockdown motoneurons (Fig. 4b). In contrast, transcripts upregulated in the axonal compartment upon loss of Tdp- 43 showed fewer iCLIP hits in their 3'UTRs compared to unchanged or downregulated transcripts. This result suggests that Tdp- 43 mediates the transport and/or stability of mRNAs in axons of motoneurons by binding to the 3'UTR of these transcripts, and it inhibits the axonal translocation when interacting with other sites.

Loss of Tdp-43 dysregulates transcripts with mitochondrial and synapse functions in the somatodendritic compartment

Gene ontology (GO) term analysis of transcripts upregulated in the somatodendritic compartment of Tdp- 43 knockdown motoneurons revealed an enrichment of transcripts with functions in neuron projection development, synapse and cytoskeleton (Fig. 5). Among these is the transcript encoding Amyloid precursor protein (App) which has been found to be elevated in the SOD1G93A mouse model of ALS [32, 33]. App ablation improved the motor functions of SOD1G93A mice, indicating the functional relevance of increased APP in ALS [33]. The most strongly upregulated transcript encodes for thioredoxin interacting protein (Txnip) (Supplementary Table 3), which is an inhibitor of thioredoxin function. Thioredoxin acts as an antioxidant such that upregulation of Txnip is expected to disturb mitochondrial function and to inhibit pathways that protect neurons against reactive oxygen species (ROS) [34]. We also detected an upregulation of the Igflr transcript encoding the IGF-1 receptor in agreement with previous studies reporting increased levels of IGF-1 receptor in the spinal cord of ALS patients [35, 36]. Furthermore, we found a significant upregulation of cAMP-specific $3^{\prime}, 5^{\prime}$-cyclic phosphodiesterase 4D (Pde4d), which hydrolyzes the second messenger cAMP. Reduced cAMP levels are expected to affect many functions related to neuronal survival and activity. Efforts to inhibit this enzyme have found their way into clinical trials for treatment of ALS (ClinicalTrials.gov, Identifier: NCT02238626), indicating that this upregulation could contribute to neuronal dysfunction and cell death in Tdp-43-depleted motoneurons.

In contrast, the group of transcripts downregulated in the somatodendritic compartment of Tdp-43-depleted motoneurons showed a high association with GO terms related to mitochondrial function and energy production
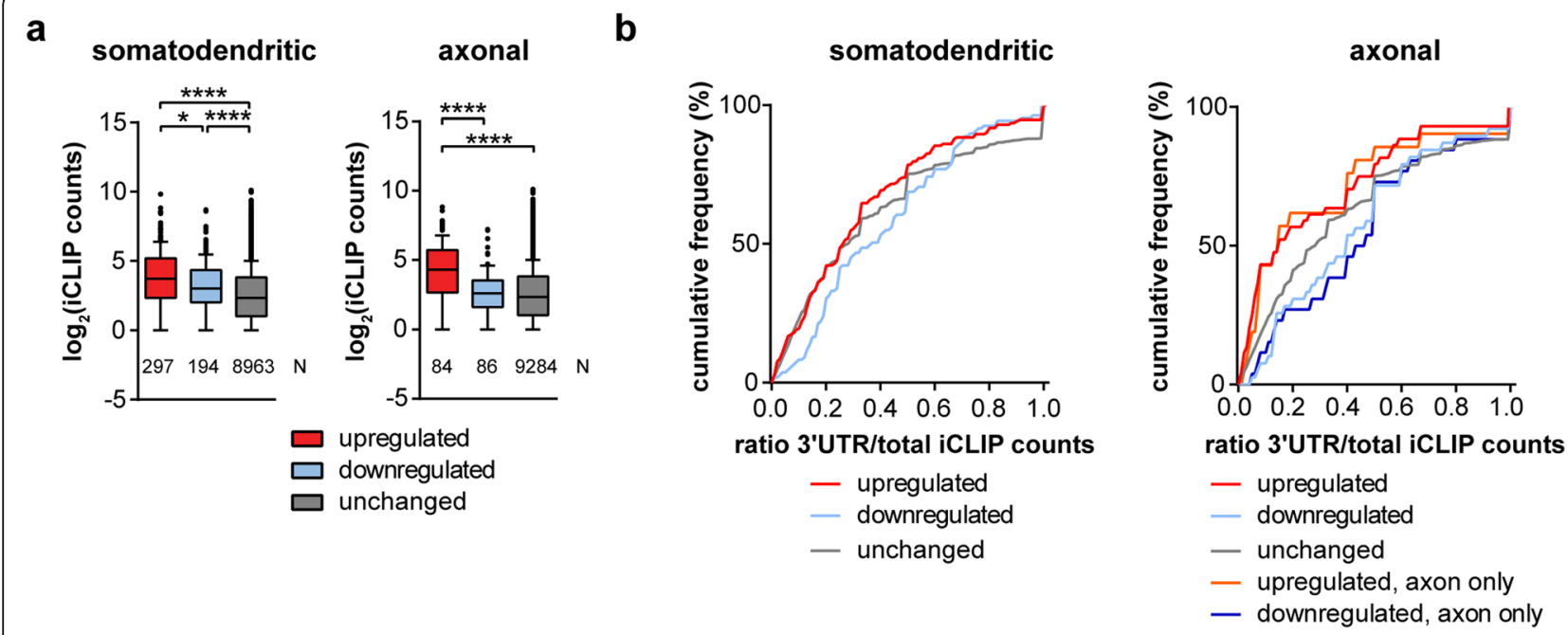

Fig. 4 Analysis of TDP-43 binding to transcripts dysregulated in Tdp-43 knockdown motoneurons. a Boxplot showing the number of TDP-43 iCLIP counts [18] for upregulated, downregulated and unchanged transcripts in the somatodendritic and axonal compartment after Tdp-43 knockdown. Only transcripts with at least one iCLIP hit were included. ${ }^{*} p \leq 0.05,{ }^{* * *} p \leq 0.0001$; Kruskal-Wallis test with Dunn's multiple comparisons test ( $N$ numbers of transcripts are shown in the graph). $\mathbf{b}$ Cumulative distribution diagram of the ratio of iCLIP hits in the $3^{\prime} U T R$ to the total number of hits. Only transcripts with at least one iCLIP hit in the $3^{\prime} U T R$ were included. Transcripts selectively altered in axons of Tdp-43 knockdown motoneurons are labeled "axon only" 


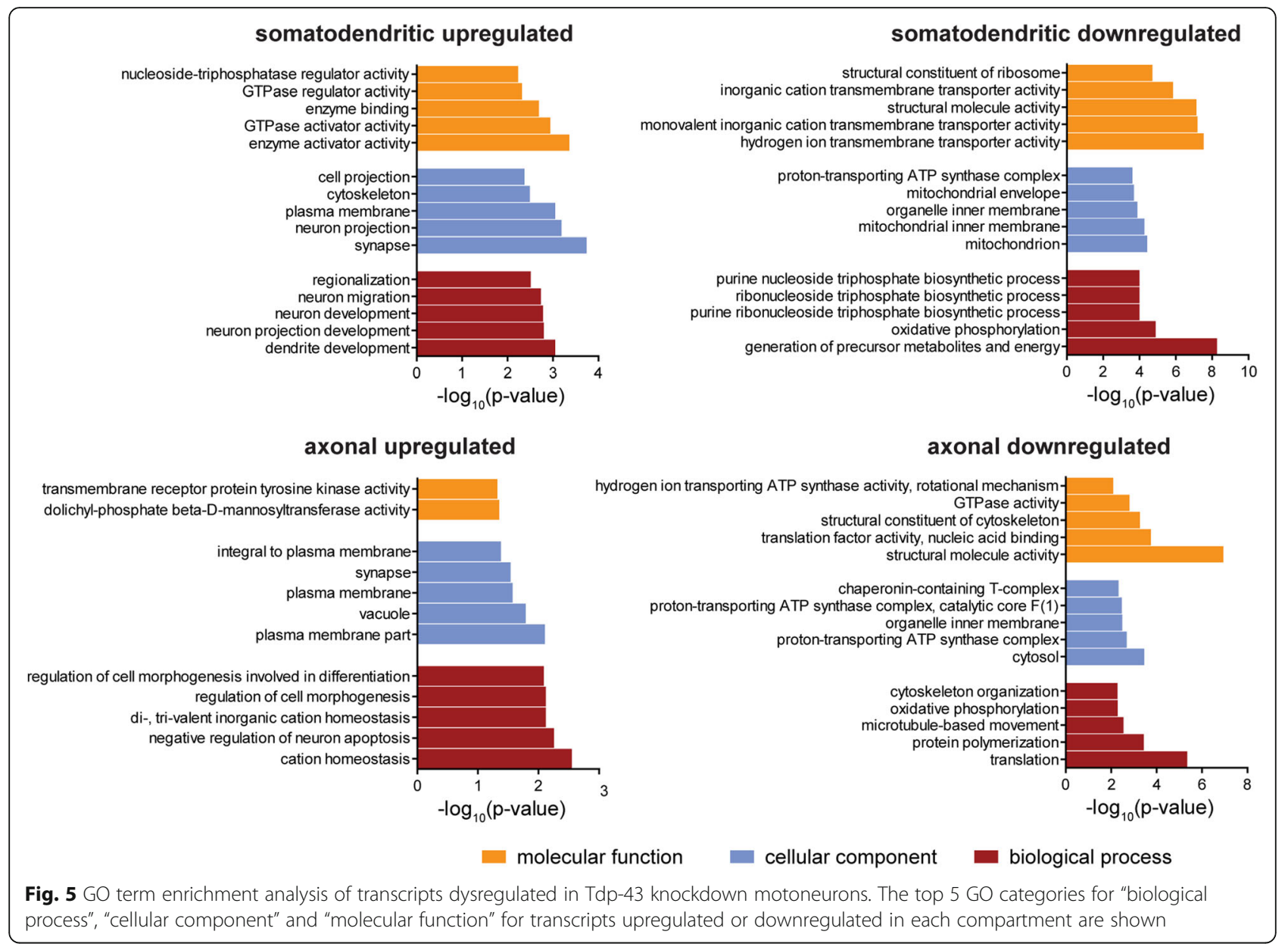

(Fig. 5). Among the most strongly downregulated genes, we found the transcripts encoding Acetyl-CoA acetyltransferase 1 (Acat1) and Cytochrome b-c1 complex subunit 8 (Uqcrq) (Supplementary Table 4). Notably, a number of transcripts encoding proteins previously linked to ALS also appeared downregulated in Tdp-43 knockdown motoneurons including the transcripts for Sod1 [37], Hnrnpa2b1 [38] and Matr3 [39].

\section{Reduced capacity for protein production in axons of Tdp- 43 knockdown motoneurons}

For transcripts downregulated in axons of Tdp- 43 knockdown motoneurons the GO term "translation" is the most significantly affected biological process (Fig. 5). The transcripts falling within this category include translation initiation factors and ribosomal proteins. For example, transcripts encoding the $40 \mathrm{~S}$ ribosomal proteins S8 (Rps8) and S3 (Rps3) and the transcript for eukaryotic initiation factor 4A-II (Eif4a2) were among the top 20 downregulated transcripts in the axonal compartment upon Tdp-43 depletion (Supplementary Table 6). The whole transcriptome profiling method we used for RNA-seq also monitors non-coding RNAs including
rRNAs [22]. We found a significant reduction in the number of reads mapping to rRNA in axons of Tdp- 43 knockdown motoneurons compared to controls while rRNA levels in the somatodendritic compartment were unchanged (Fig. 6a). Based on these results we investigated the possibility that Tdp-43 deficiency impairs protein translation in axons. We set up a puromycin labeling assay in which motoneurons were cultured for 6 $\mathrm{d}$ and then exposed to puromycin for $15 \mathrm{~min}$. Puromycin is incorporated into nascent polypeptide chains and can be detected by immunostaining with a puromycinspecific antibody. Following puromycin exposure, wildtype motoneurons showed prominent immunolabeling in the cytosol of the soma as well as in axons (Fig. 6b). Omission of puromycin or pre-treatment with the protein synthesis inhibitor anisomycin strongly reduced the immunosignal, indicating specificity of the puromycin labeling assay. The axonally localized puromycin immunoreactivity most likely originated from proteins that were locally synthesized in axons, because the $15 \mathrm{~min}$ pulse is too short to allow anterograde transport of these proteins from the cell body. To test this possibility in more detail, we exposed motoneurons to nocodazole, an 
a

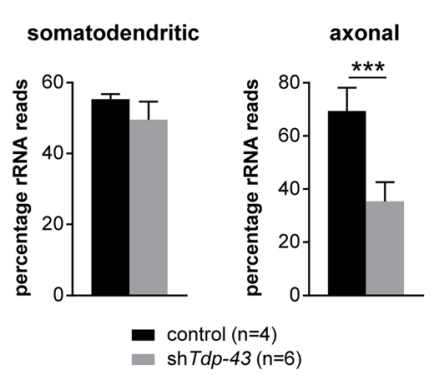

b
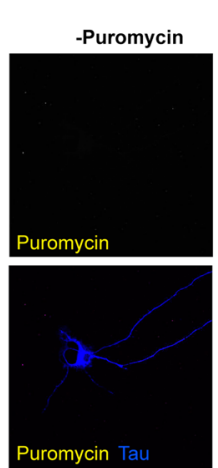
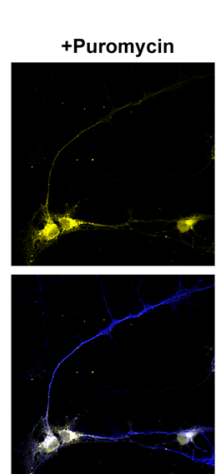

+Anisomycin
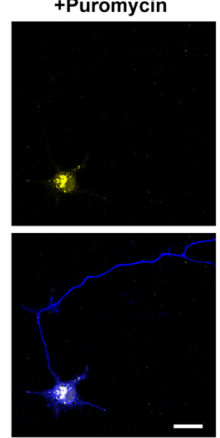

c

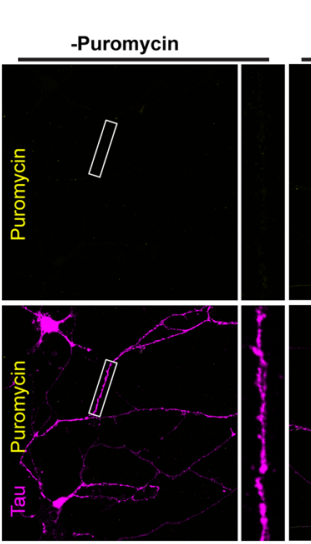

d

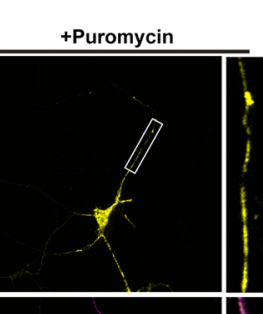
+Nocodazole
+Puromycin +Cycloheximide
+ Puromycin

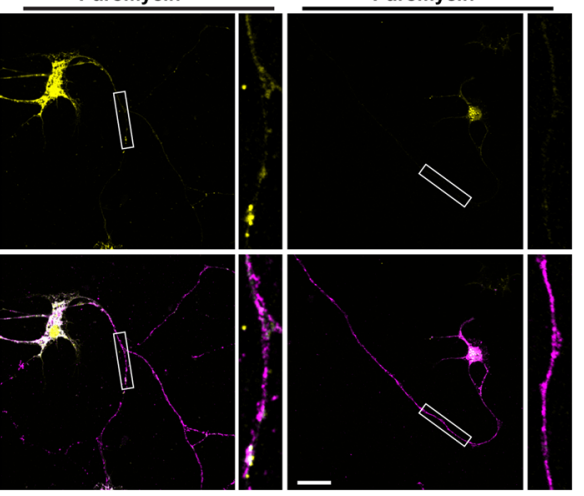

+Puromycin e
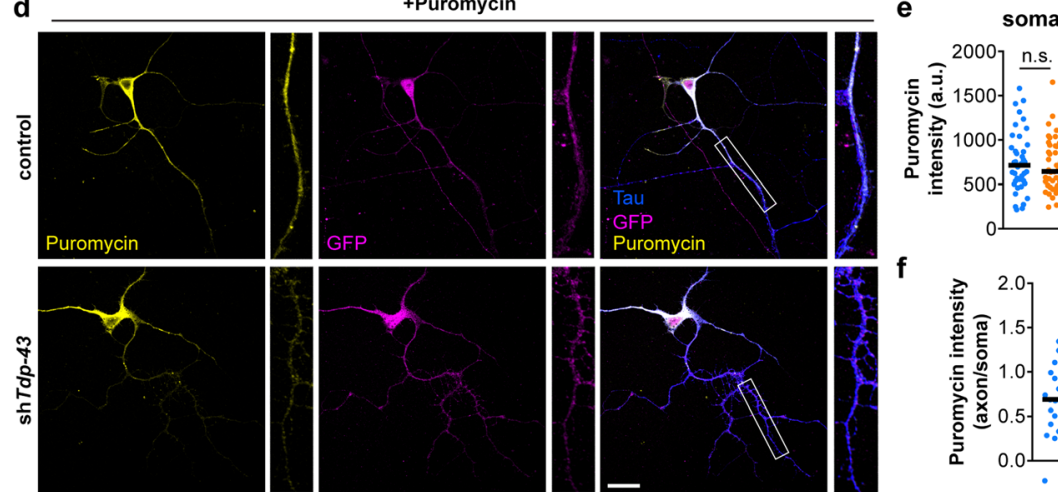

axon

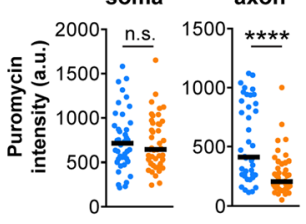

f
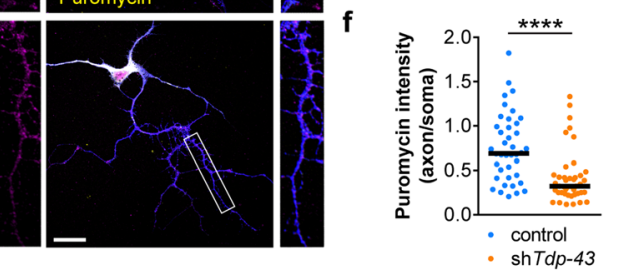

Fig. 6 Reduced protein synthesis in axons of Tdp-43 knockdown motoneurons. a Percentage of reads mapping to rRNAs from RNA-seq data of Tdp-43 knockdown (shTdp-43) and control motoneurons. Data represent mean \pm SD. ${ }^{* * *} p \leq 0.001$; unpaired t-test. $\mathbf{b}$ Immunostaining of cultured motoneurons with antibodies against puromycin and Tau. Motoneurons were exposed to puromycin as indicated. Treatment with anisomycin was done prior to puromycin exposure. Scale bar: $20 \mu \mathrm{m}$. c Motoneurons were exposed to nocodazole or cycloheximide as indicated followed by puromycin labeling. Immunostaining was performed with antibodies against puromycin and Tau. Boxes indicate magnified axon segments. Scale bar: $20 \mu \mathrm{m}$. d Immunostaining of control and Tdp-43 knockdown motoneurons with antibodies against puromycin and Tau. Motoneurons were transduced with control or Tdp-43 knockdown lentivirus and cultured for $6 \mathrm{~d}$ followed by puromycin labeling. Boxes indicate magnified axon segments. Scale bar: $20 \mu \mathrm{m}$. e Scatter plots showing fluorescence intensity of the puromycin immunosignal in somata and axons of Tdp-43 knockdown and control motoneurons. Horizontal lines represent median. n.s. not significant, ${ }^{* * *} p \leq 0.0001 ;$ Mann-Whitney test $(\mathrm{N}=40$ motoneurons for control and 43 motoneurons for Tdp-43 knockdown motoneurons, $n=3$ independent experiments). $\mathbf{f}$ Scatter plot showing the axon-to-soma ratios of puromycin intensity in control and Tdp-43 knockdown motoneurons. Horizontal lines represent median. ${ }^{* * * *} p \leq 0.0001$; Mann-Whitney test ( $N=40$ motoneurons for control and 43 motoneurons for Tdp-43 knockdown motoneurons, $n=3$ independent experiments)

inhibitor of microtubule assembly that disrupts axonal transport [40], prior to puromycin labeling. Following nocodazole treatment, puromycin immunoreactivity was still detectable in axons of motoneurons (Fig. 6c).
Treatment with the translation inhibitor cycloheximide prior to puromycin exposure abolished puromycin immunolabeling indicating signal specificity. Thus, puromycin immunoreactivity in axons of motoneurons reflects local 
protein synthesis that can occur in the absence of anterograde axonal transport. Next, we investigated puromycin incorporation in Tdp-43 knockdown motoneurons (Fig. $6 \mathrm{~d})$. This analysis showed that puromycin immunolabeling was exclusively reduced in axons but not in somata of Tdp-43 knockdown motoneurons relative to controls (Fig. 6e) resulting in a significantly reduced ratio of axonal to somatic puromycin labeling intensity upon Tdp-43 depletion (Fig. 6f). Thus, Tdp-43-depleted motoneurons have a reduced capacity for protein synthesis in their axons.

\section{Disturbed mitochondrial functionality in Tdp-43-deficient axons}

Beyond transcripts related to translation, we found an enrichment of GO terms related to mitochondrial functions for transcripts downregulated in the axonal compartment of Tdp-43 knockdown motoneurons (Fig. 5).
These GO terms contain the transcript encoding the mitochondrial ATP synthase beta-subunit (Atp5b) which has previously been associated with ALS [41]. In order to assess whether axonal mitochondria are affected by Tdp-43 depletion, we labeled mitochondria in control and Tdp-43 knockdown motoneurons with MitoTracker $^{\text {re }}$ Orange. We found that the number of intact mitochondria was significantly reduced in axons of Tdp43 knockdown motoneurons relative to controls (Fig. 7a). Next, we assessed the mitochondrial membrane potential using the fluorescent dye tetramethylrhodamine (TMRM). As a negative control we used the uncoupling agent FCCP, which depolarizes the mitochondrial membrane potential. Treatment with FCCP led to rapid loss of the TMRM signal indicating the specificity of the labeling procedure (Fig. $7 \mathrm{~b}$ ). We then labeled control and Tdp-43 knockdown motoneurons with TMRM (Fig. 7c).

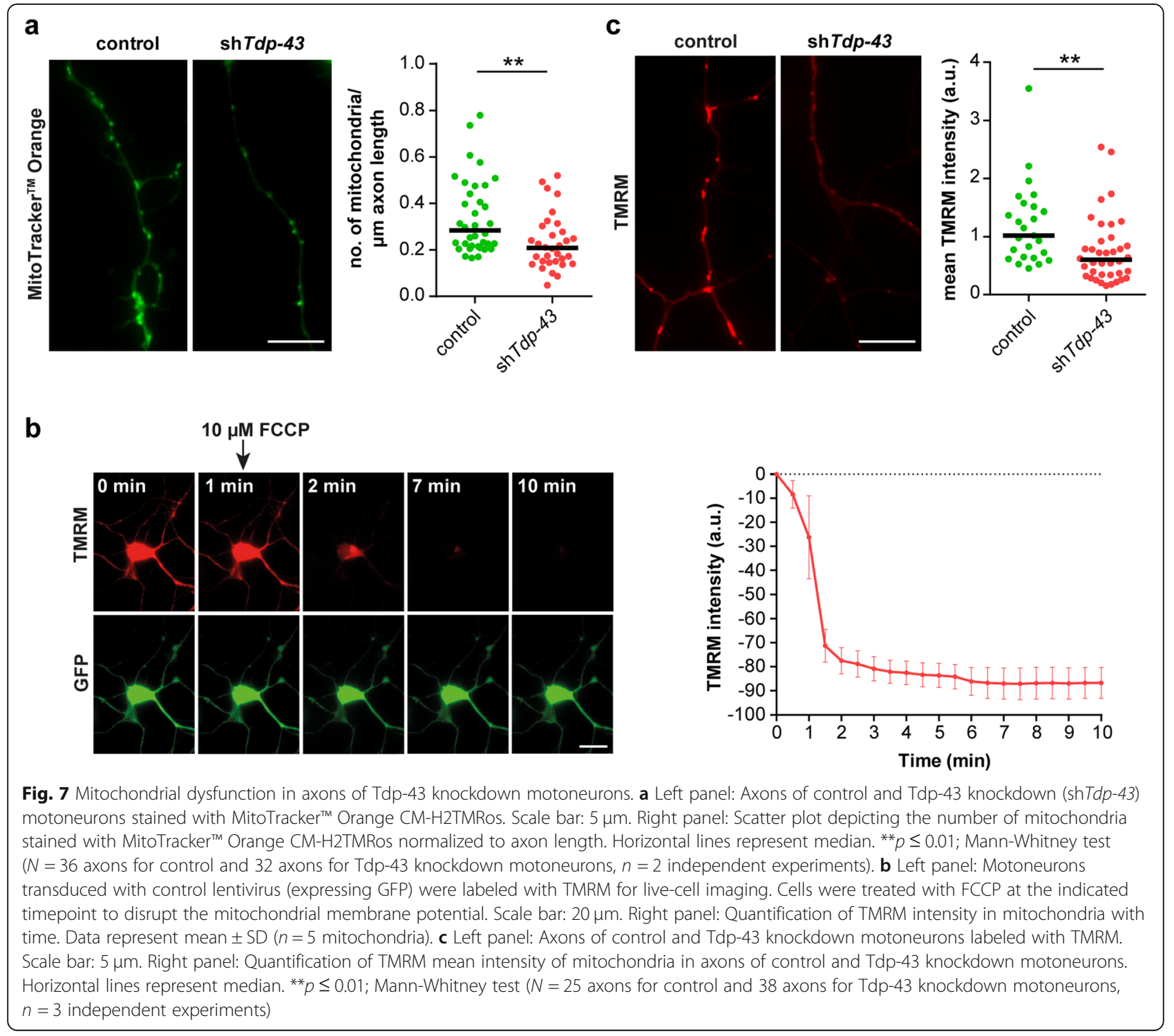


This analysis revealed that the mean labeling intensity of mitochondria was significantly reduced in axons of Tdp43 knockdown motoneurons relative to controls. Thus, Tdp-43 depletion affects the number and functionality of axonal mitochondria.

\section{Nicotinamide treatment rescues axonal defects of Tdp-43- deficient motoneurons}

Our data point towards the possibility that a lack of energy supply due to disturbed mitochondrial function contributes to the axon growth defect associated with Tdp-43 depletion in motoneurons. To investigate this possibility we treated Tdp-43 knockdown and control motoneurons with nicotinamide (NAM), a precursor for nicotinamide adenine dinucleotide (NAD+) that acts as a key cofactor in mitochondrial pathways for the production of ATP. NAM is converted to NAD+ through the salvage pathway [42]. We found that treatment with $0.1 \mathrm{mM}$ NAM restored axon growth of Tdp-43 knockdown motoneurons to normal levels, while axons of control motoneurons were not altered by exposure to NAM (Fig. 8a). The same rescue effect was observed with NAM concentrations up to $1 \mathrm{mM}$.

We then tested whether NAM treatment had an impact on the defective axonal protein synthesis which we observed upon Tdp-43 depletion. Control and Tdp-43 knockdown motoneurons were cultured in the presence or absence of $0.1 \mathrm{mM}$ NAM for $6 \mathrm{~d}$ followed by puromycin exposure to label nascent proteins (Fig. 8b). In the absence of NAM, puromycin immunolabeling was reduced in axons of Tdp- 43 knockdown motoneurons relative to control motoneurons (Fig. 8b, c). Treatment with NAM significantly increased puromycin immunoreactivity in axons of Tdp-43 knockdown motoneurons relative to axons of untreated Tdp-43 knockdown motoneurons (Fig. 8b, c). Thus, NAM exposure rescued the axon growth defect of motoneurons depleted of Tdp-43 and increased their capacity for axonal protein synthesis.

\section{Discussion}

In this study, we have investigated transcriptome alterations in the somatodendritic and axonal compartments of motoneurons after Tdp-43 depletion. Subcellular transcriptome alterations in ALS cell models have been investigated before using different approaches. In the study by Rotem et al. mouse motoneurons were transduced with lentiviruses for expression of GFP-tagged SOD1(G93A) or TDP-43(A315T) and cultured in modified Boyden chambers to obtain neuritic RNA [43]. Nijssen et al. developed an optimized procedure for conducting axonal RNA-seq on motoneurons grown in microfluidic chambers and used this technique to profile axons of mouse embryonic stem cell (mESC)-derived motoneurons overexpressing SOD1(G93A) [44]. While these studies revealed candidate transcripts whose dysregulation might contribute to motoneuron dysfunction in ALS, it needs to be considered that overexpression of SOD1 or TDP-43 might induce deleterious side effects in a dose-dependent manner [45].

It has been suggested that, in addition to the toxicity exerted by TDP- 43 insoluble inclusions, loss of TDP-43 functions contributes to the etiology of ALS [46]. Given that TDP-43 ALS mutants show reduced motility in axons [4] and that axonal degeneration precedes motoneuron loss in ALS mouse models [47, 48], loss of axonal TDP-43 functions might be an early driving factor contributing to the motoneuron pathology underlying ALS. Therefore, to investigate the effects of Tdp- 43 depletion on the composition of subcellular transcriptomes we cultured Tdp-43 knockdown motoneurons in microfluidic chambers and used a whole transcriptome profiling method for identification of RNA alterations. Major changes were detectable in the somatodendritic as well as the axonal compartment and similar numbers of transcripts were up- or downregulated. These deregulations might disturb multiple pathways and mechanisms that are relevant for neuronal survival and axonal maintenance as well as for synaptic function and integrity. In agreement with this notion, we observed several defects in axons of Tdp-43-deficient motoneurons, including loss of transcripts encoding cytoskeletal proteins, reduced protein synthesis and disturbed mitochondrial function. In addition to transcripts encoding proteins important for motoneuron biology, we observed that the axonal localization also of non-polyadenylated RNAs such as ribosomal RNAs is affected by Tdp- 43 depletion. Therefore, our results suggest that the pathology induced by Tdp- 43 deficiency cannot simply be explained by one individual key mechanism, but rather by a combination of defects that play together and contribute to a complex disease phenotype.

After Tdp-43 depletion, several transcripts are upregulated in the soma that appear highly relevant for neuronal integrity. For example, upregulation of Txnip is expected to result in an inhibition of thioredoxin leading to enhanced oxidative stress. The Pi3K/AKT pathway, which is important for neuronal survival [49] and proper mitochondrial function [50], modulates Txnip expression and thereby protects cells against ROS [51]. AKTmediated inhibition of the transcription factor FOXO1 can reduce Txnip levels [51]. This then leads to enhanced thioredoxin activity, which is necessary for ROS detoxification. The observations that FOXO1 represses TXNIP transcription directly $[52,53]$, and that TDP-43 controls FOXO activity [54] indicate that this pathway might contribute to ALS pathology. The enhanced expression of Txnip we observed after Tdp-43 depletion could cause inhibition of thioredoxin activity, leading to 

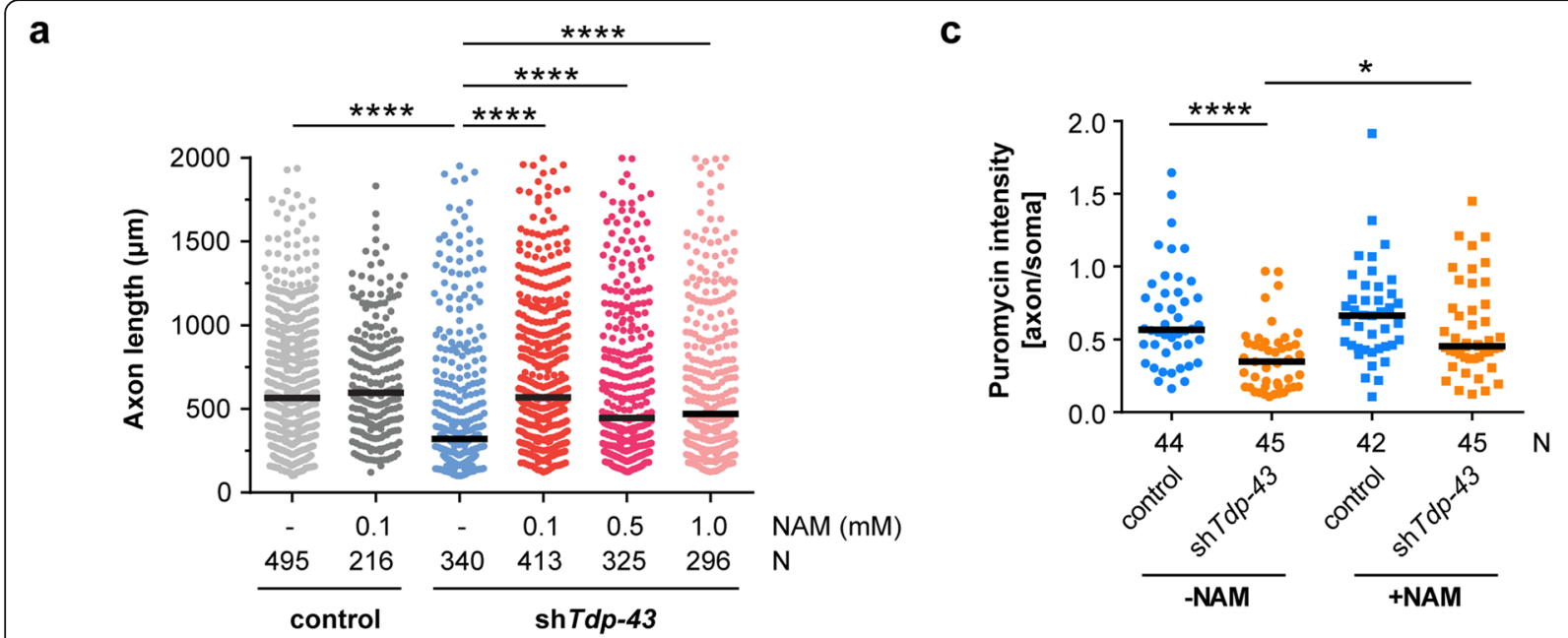

b
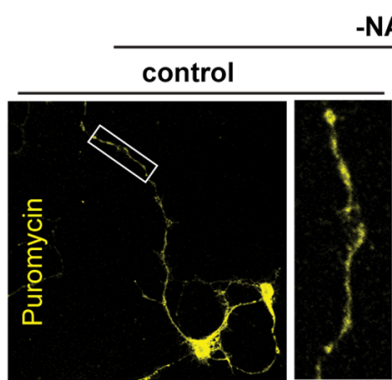

NAM
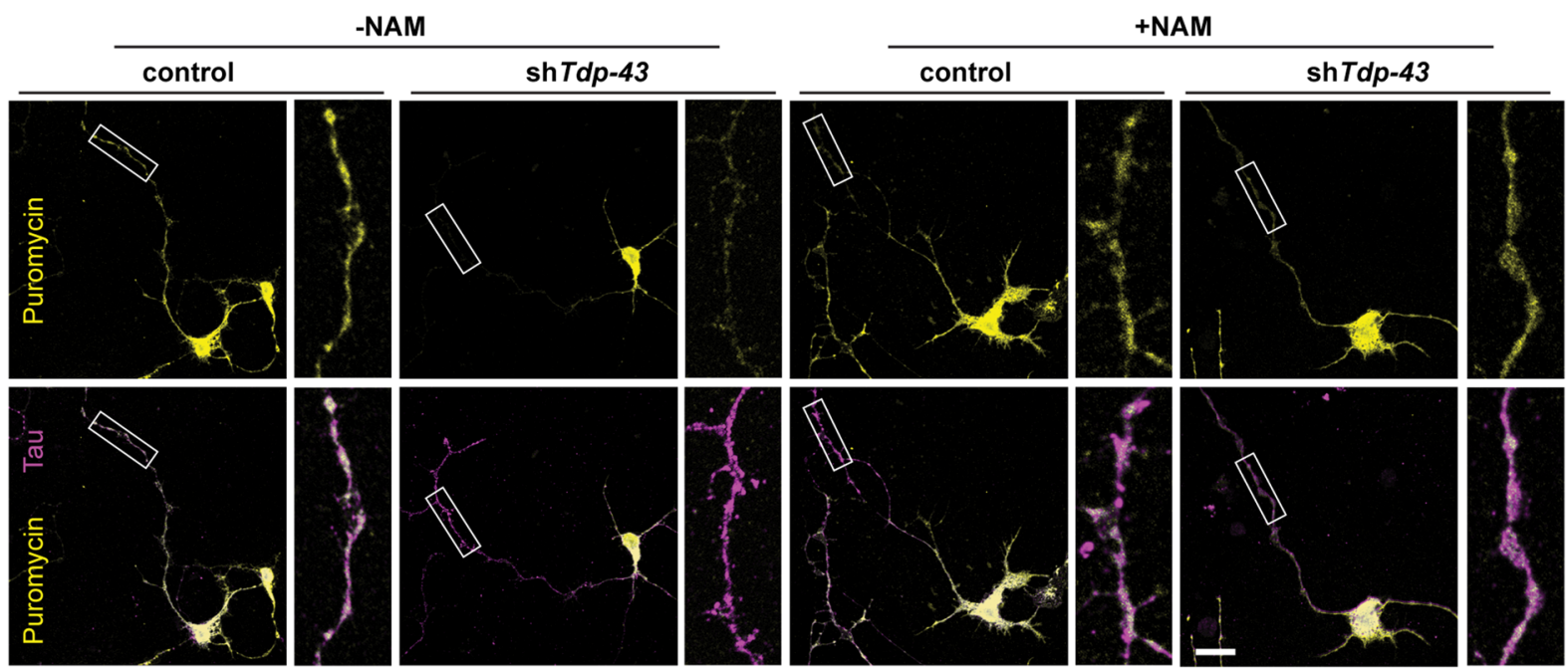

Fig. 8 Nicotinamide (NAM) rescues axon defects of Tdp-43 knockdown motoneurons. a Scatter plot of axon lengths of Tdp-43 knockdown (shTdp-43) and control motoneurons cultured in the absence (-) or presence of NAM at the indicated concentrations. Horizontal lines represent median. ${ }^{* * *} p \leq 0.0001$; Kruskal-Wallis test with Dunn's multiple comparisons test ( $N$ numbers of axons are shown in the graph, $n=3$ independent experiments). b Immunostaining of control and Tdp-43 knockdown motoneurons with antibodies against puromycin and Tau. Motoneurons were transduced with control or Tdp-43 knockdown lentivirus and cultured in the presence (+) or absence (-) of 0.1 mM NAM. Puromycin labeling was performed at DIV6. Scale bar: $20 \mu \mathrm{m}$. c Scatter plot showing the axon-to-soma ratios of puromycin intensity in control and Tdp-43 knockdown motoneurons. Horizontal lines represent median. ${ }^{*} p \leq 0.05,{ }^{* * *} p \leq 0.0001$; Kruskal Wallis test with Dunn's multiple comparisons test ( $\mathrm{N}$ numbers of motoneurons are shown in the graph, $n=3$ independent experiments)

enhanced ROS levels. This, in turn, could induce the observed mitochondrial dysfunction and thus contribute to the pathological alterations in mitochondria seen in Tdp-43-associated motoneuron disease models. The rescue effect by treatment with NAM, which has been shown to reduce ROS levels [55], supports this hypothesis and indicates that this pathway could represent a central part of the pathology in ALS and other diseases in which TDP-43 loss of function is apparent.

GO term analysis of transcripts downregulated in the axonal compartment of Tdp-43-deficient motoneurons revealed that pathways related to mitochondrial functions might be particularly affected. Mitochondria are a known target of Tdp-43 function [26, 27, 56-60] and are also defective in iPS-cell derived motoneurons harboring ALS mutations [61] and ALS mouse models [62], indicating that defects in energy production upon loss of Tdp- 43 might contribute to the axonal growth defect in cultured motoneurons. In agreement with this notion, we found a decreased number and activity of mitochondria in axons of Tdp-43 knockdown motoneurons. Moreover, treatment with the NAD+ precursor NAM restored axon growth of Tdp-43-depleted motoneurons to near-normal levels. This suggests that reduced energy levels might play an 
important role in the axonal pathology upon Tdp-43 loss. However, besides energy support, NAM can also exert regulatory functions outside mitochondria. For example, Sirtuins (SIRTs) are protein deacetylases that use NAD+ as a cofactor and convert it to NAM during the deacetylation reaction [63]. NAM itself acts as an inhibitor of Sirtuin activities. Inhibition of SIRT2 has been found to be neuroprotective in models of Parkinson's disease and other neurodegenerative disease contexts $[64,65]$. Therefore, dysregulation of Sirtuins might contribute to the axonal defects we observed in Tdp-43 knockdown motoneurons. Recently, the administration of NAM has been shown to improve motor symptoms of SOD1G93A mice [66]. Furthermore, reduced NAM levels have been observed in ALS patients [66]. Thus, NAM and associated downstream pathways might represent potential avenues for new ALS treatment options.

We found that reduced axon growth is a key morphological phenotype of Tdp-43-deficient motoneurons. We observed this phenotype with three different shRNAs targeting the $T d p-43$ transcript. The reduced axon lengths we observed for Tdp-43-depleted motoneurons stand in contrast to previous observations [25], but our data are in agreement with other studies investigating neuronal defects upon TDP-43 reduction [67, 68]. Beyond that, expression of TDP-43 mutants harboring ALS mutations also leads to neurite growth defects [69]. Reduced axon growth is seen in many pathologies relevant to motoneuron diseases. In the case of SMN protein deficiency which causes SMA, reduced axon growth accompanied by deficits in presynaptic actin synthesis and excitability were observed in mouse models [70-73]. In motoneurons depleted of C9orf72 as a cell culture model of ALS, dysregulation of actin dynamics in axons appears prominent [74]. This raises the question whether similar or distinct mechanisms are responsible for defective axon growth in Tdp-43-depleted motoneurons. Our data show that multiple pathways are affected by loss of Tdp-43. On the one side, mitochondrial dysfunction and enhanced ROS levels are expected to reduce cellular metabolism and thus also axon growth. On the other side, we found dysregulation of transcripts related to protein translation in the axonal compartment of Tdp-43 knockdown motoneurons. Also, rRNA levels were significantly reduced in the axonal but not somatodendritic compartment of Tdp-43 knockdown motoneurons. In agreement, we found that Tdp-43-depleted motoneurons have a reduced capacity for axonal protein synthesis, which might contribute to defective axon growth. Treatment with NAM partially rescued this defect, indicating that pathways related to energy metabolism can alleviate or delay pathological processes in axons of motoneurons with loss of Tdp-43 function.
In summary, our data have revealed that multiple pathways are dysregulated in Tdp-43-depleted motoneurons, many of them having been characterized before in the context of other neurodegenerative diseases such as Alzheimer's, Huntington's and Parkinson's disease. Thus, it might be the combination of defects in these pathways that underlies the vulnerability and axonal degeneration of Tdp-43-deficient motoneurons. A multitude of targets could be conferred from these pathways that might need to be addressed in combination for therapy development in ALS.

\section{Supplementary information}

Supplementary information accompanies this paper at https://doi.org/10. 1186/s40478-020-00987-6.

Additional file 1: Supplementary Table 1. List of primers for cloning of shRNAs into $\mathrm{pSIH}$. siRNA sequences are underlined. Supplementary Table 2. List of $q P C R$ primers. Supplementary Table 3. List of transcripts significantly $(p<0.05)$ upregulated in the somatodendritic compartment of Tdp-43 knockdown (T_sd) motoneurons relative to control (G_sd) motoneurons. Supplementary Table 4. List of transcripts sig nificantly $(p<0.05)$ downregulated in the somatodendritic compartment of Tdp-43 knockdown ( $T$ s sd) motoneurons relative to control (G_sd) motoneurons. Supplementary Table $\mathbf{5}$. List of transcripts significantly $(p<$ 0.05) upregulated in the axonal compartment of Tdp-43 knockdown (T_ax) motoneurons relative to control (G_ax) motoneurons. Supplementary Table 6. List of transcripts significantly $(p<0.05)$ downregulated in the axonal compartment of Tdp-43 knockdown (T_ax) motoneurons relative to control (G_ax) motoneurons. Supplementary Table 7. List of transcripts significantly $(p<0.05)$ deregulated in the somatodendritic (sd) and axonal (ax) compartment of Tdp-43 knockdown motoneurons relative to control motoneurons.

\section{Acknowledgements}

We thank C. Drepper for helpful discussions.

\section{Authors' contributions}

$M B, L S B$ and MS conceived the project, designed the experiments and wrote the manuscript with the assistance of all co-authors. MB performed RNA-seq library preparation and data analysis. LSB performed compartmentalized motoneuron cultures, qPCR and axon length measurements. PL performed puromycin labeling assays. MM and CD performed mitochondrial measurements and immunofluorescence staining of motoneurons. BD performed immunofluorescence staining of spinal cord sections. VS and SJ tested Tdp-43 knockdown viruses by qPCR and performed axon length measurements. SA performed bioinformatic analysis of RNA-seq data. All authors read and approved the final manuscript.

\section{Funding}

This work was supported by the Deutsche Forschungsgemeinschaft [BR4910/ 1-1 (SPP1738) and BR4910/2-1 (SPP1935) to M.B., SE697/4-1 (SPP1738), SE697/5-1 (SPP1935) and SE697/1 to M.S.]. This publication was supported by the Open Access Publication Fund of the University of Wuerzburg.

\section{Availability of data and materials}

The sequencing data described in this publication are accessible in NCBI's Gene Expression Omnibus through GEO Series accession number GSE147607.

\section{Ethics approval and consent to participate}

Experiments involving animals were conducted following the regulations on animal protection of the German federal law and of the Association for Assessment and Accreditation of Laboratory Animal Care, in agreement with and under control of the local veterinary authority. 


\section{Consent for publication}

Not applicable.

\section{Competing interests}

The authors declare that they have no competing interests.

\section{Author details}

${ }^{1}$ Institute of Clinical Neurobiology, University of Wuerzburg, 97078 Wuerzburg, Germany. ${ }^{2}$ Comprehensive Cancer Center Mainfranken, University of Wuerzburg, 97080 Wuerzburg, Germany.

\section{Received: 3 July 2020 Accepted: 4 July 2020}

\section{Published online: 24 July 2020}

\section{References}

1. Briese M, Saal-Bauernschubert L, Ji C, Moradi M, Ghanawi H, Uhl M et al (2018) hnRNP R and its main interactor, the noncoding RNA 7SK, coregulate the axonal transcriptome of motoneurons. Proc Natl Acad Sci U S A 115: E2859-E2868. https://doi.org/10.1073/pnas.1721670115

2. Cestra G, Rossi S, Di SM, Cozzolino M (2017) Control of mRNA translation in ALS Proteinopathy. Front Mol Neurosci 10:85. https://doi.org/10.3389/fnmol. 2017.00085

3. Lehmkuhl EM, Zarnescu DC (2018) Lost in translation: evidence for protein synthesis deficits in ALS/FTD and related neurodegenerative diseases. Adv Neurobiol 20:283-301. https://doi.org/10.1007/978-3-319-89689-2 11

4. Alami NH, Smith RB, Carrasco MA, Williams LA, Winborn CS, Han SS et al (2014) Axonal transport of TDP-43 mRNA granules is impaired by ALScausing mutations. Neuron 81:536-543. https://doi.org/10.1016/j.neuron. 2013.12.018

5. Barmada SJ (2015) Linking RNA dysfunction and neurodegeneration in amyotrophic lateral sclerosis. Neurotherapeutics 12:340-351. https://doi.org/ 10.1007/s13311-015-0340-3

6. Gershoni-Emek N, Chein M, Gluska S, Perlson E (2015) Amyotrophic lateral sclerosis as a spatiotemporal mislocalization disease: location, location, location. Int Rev Cell Mol Biol 315:23-71. https://doi.org/10.1016/bs.ircmb. 2014.11.003

7. Saal L, Briese M, Kneitz S, Glinka M, Sendtner M (2014) Subcellular transcriptome alterations in a cell culture model of spinal muscular atrophy point to widespread defects in axonal growth and presynaptic differentiation. RNA 20:1789-1802. https://doi.org/10.1261/rna.047373.114

8. Ou SH, Wu F, Harrich D, Garcia-Martinez LF, Gaynor RB (1995) Cloning and characterization of a novel cellular protein, TDP-43, that binds to human immunodeficiency virus type 1 TAR DNA sequence motifs. J Virol 69:35843596 https://jvi.asm.org/content/69/6/3584.long

9. Buratti E, Baralle FE (2010) The multiple roles of TDP-43 in pre-mRNA processing and gene expression regulation. RNA Biol 7:420-429. https://doi. org/10.4161/rna.7.4.12205

10. Cohen TJ, Lee VM, Trojanowski JQ (2011) TDP-43 functions and pathogenic mechanisms implicated in TDP-43 proteinopathies. Trends Mol Med 17:659667. https://doi.org/10.1016/j.molmed.2011.06.004

11. Ratti A, Buratti E (2016) Physiological functions and pathobiology of TDP-43 and FUS/TLS proteins. J Neurochem 138(Suppl 1):95-111. https://doi.org/10. 1111/jnc.13625

12. Ayala YM, Zago P, D'Ambrogio A, Xu YF, Petrucelli L, Buratti E et al (2008) Structural determinants of the cellular localization and shuttling of TDP-43. J Cell Sci 121:3778-3785. https://doi.org/10.1242/jcs.038950

13. Brown RH, Al-Chalabi A (2017) Amyotrophic lateral sclerosis. N Engl J Med 377:162-172. https://doi.org/10.1056/NEJMra1603471

14. Yasuda K, Mili S (2016) Dysregulated axonal RNA translation in amyotrophic lateral sclerosis. Wiley Interdiscip Rev RNA 7:589-603. https://doi.org/10. 1002/wrna.1352

15. Baldwin KR, Godena VK, Hewitt VL, Whitworth AJ (2016) Axonal transport defects are a common phenotype in Drosophila models of ALS. Hum Mol Genet 25:2378-2392. https://doi.org/10.1093/hmg/ddw105

16. Polymenidou M, Lagier-Tourenne C, Hutt KR, Huelga SC, Moran J, Liang TY et al (2011) Long pre-mRNA depletion and RNA missplicing contribute to neuronal vulnerability from loss of TDP-43. Nat Neurosci 14:459-468. https:// doi.org/10.1038/nn.2779

17. Sephton CF, Cenik C, Kucukural A, Dammer EB, Cenik B, Han Y et al (2011) Identification of neuronal RNA targets of TDP-43-containing ribonucleoprotein complexes. J Biol Chem 286:1204-1215. https://doi.org/ 10.1074/jbc.M110.190884

18. Tollervey JR, Curk T, Rogelj B, Briese M, Cereda M, Kayikci M et al (2011) Characterizing the RNA targets and position-dependent splicing regulation by TDP-43. Nat Neurosci 14:452-458. https://doi.org/10.1038/nn.2778

19. Wiese S, Herrmann T, Drepper C, Jablonka S, Funk N, Klausmeyer A et al (2010) Isolation and enrichment of embryonic mouse motoneurons from the lumbar spinal cord of individual mouse embryos. Nat Protoc 5:31-38. https://doi.org/10.1038/nprot.2009.193

20. Subramanian N, Wetzel A, Dombert B, Yadav P, Havlicek S, Jablonka S et al (2012) Role of $\mathrm{Na}(\mathrm{v}) 1.9$ in activity-dependent axon growth in motoneurons. Hum Mol Genet 21:3655-3667. https://doi.org/10.1093/hmg/dds195

21. Luningschror $P$, Binotti B, Dombert B, Heimann P, Perez-Lara A, Slotta C et al (2017) Plekhg5-regulated autophagy of synaptic vesicles reveals a pathogenic mechanism in motoneuron disease. Nat Commun 8:678. https://doi.org/10.1038/s41467-017-00689-z

22. Briese M, Saal L, Appenzeller S, Moradi M, Baluapuri A, Sendtner M (2016) Whole transcriptome profiling reveals the RNA content of motor axons. Nucleic Acids Res 44:e33. https://doi.org/10.1093/nar/gkv1027

23. Edgar R, Domrachev M, Lash AE (2002) Gene expression omnibus: NCBI gene expression and hybridization array data repository. Nucleic Acids Res 30:207-210 https://doi.org/10.1093/nar/30.1.207

24. Huang dW, Sherman BT, Lempicki RA (2009) Systematic and integrative analysis of large gene lists using DAVID bioinformatics resources. Nat Protoc 4:44-57. https://doi.org/10.1038/nprot.2008.211

25. Fallini C, Bassell GJ, Rossoll W (2012) The ALS disease protein TDP-43 is actively transported in motor neuron axons and regulates axon outgrowth. Hum Mol Genet 21:3703-3718. https://doi.org/10.1093/hmg/dds205

26. Davis SA, Itaman S, Khalid-Janney CM, Sherard JA, Dowell JA, Cairns NJ et al (2018) TDP-43 interacts with mitochondrial proteins critical for mitophagy and mitochondrial dynamics. Neurosci Lett 678:8-15. https://doi.org/10. 1016/.neulet.2018.04.053

27. Wang W, Wang L, Lu J, Siedlak SL, Fujioka H, Liang J et al (2016) The inhibition of TDP-43 mitochondrial localization blocks its neuronal toxicity. Nat Med 22:869-878. https://doi.org/10.1038/nm.4130

28. Strong MJ, Volkening K, Hammond R, Yang W, Strong W, Leystra-Lantz C et al (2007) TDP43 is a human low molecular weight neurofilament (hNFL) mRNA-binding protein. Mol Cell Neurosci 35:320-327. https://doi.org/10. 1016/j.mcn.2007.03.007

29. Bergeron C, Beric-Maskarel K, Muntasser S, Weyer L, Somerville MJ, Percy ME (1994) Neurofilament light and polyadenylated mRNA levels are decreased in amyotrophic lateral sclerosis motor neurons. J Neuropathol Exp Neurol 53:221-230. https://doi.org/10.1097/00005072-199405000-00002

30. Oberstadt M, Classen J, Arendt T, Holzer M (2018) TDP-43 and cytoskeletal proteins in ALS. Mol Neurobiol 55:3143-3151. https://doi.org/10.1007/ s12035-017-0543-1

31. Andreassi C, Riccio A (2009) To localize or not to localize: mRNA fate is in 3'UTR ends. Trends Cell Biol 19:465-474. https://doi.org/10.1016/j.tcb.2009. 06.001

32. Rabinovich-Toidman P, Rabinovich-Nikitin I, Ezra A, Barbiro B, Fogel H, Slutsky I et al (2015) Mutant SOD1 increases APP expression and phosphorylation in cellular and animal models of ALS. PLoS One 10: e0143420. https://doi.org/10.1371/journal.pone.0143420

33. Bryson JB, Hobbs C, Parsons MJ, Bosch KD, Pandraud A, Walsh FS et al (2012) Amyloid precursor protein (APP) contributes to pathology in the SOD1(G93A) mouse model of amyotrophic lateral sclerosis. Hum Mol Genet 21:3871-3882. https://doi.org/10.1093/hmg/dds215

34. Nasoohi S, Ismael S, Ishrat T (2018) Thioredoxin-interacting protein (TXNIP) in cerebrovascular and neurodegenerative diseases: regulation and implication. Mol Neurobiol 55:7900-7920. https://doi.org/10.1007/s12035018-0917-z

35. Wilczak N, de Vos RA, De KJ (2003) Free insulin-like growth factor (IGF)-I and IGF binding proteins 2, 5, and 6 in spinal motor neurons in amyotrophic lateral sclerosis. Lancet 361:1007-1011. https://doi.org/10.1016/S01406736(03) 12828-0

36. Adem A, Ekblom J, Gillberg PG, Jossan SS, Hoog A, Winblad B et al (1994) Insulin-like growth factor-1 receptors in human spinal cord: changes in amyotrophic lateral sclerosis. J Neural Transm Gen Sect 97:73-84. https:// doi.org/10.1007/BF01277964

37. Rosen DR, Siddique $T$, Patterson D, Figlewicz DA, Sapp P, Hentati A et al (1993) Mutations in Cu/Zn superoxide dismutase gene are associated with 
familial amyotrophic lateral sclerosis. Nature 362:59-62. https://doi.org/10. 1038/362059a0

38. Kim HJ, Kim NC, Wang YD, Scarborough EA, Moore J, Diaz Z et al (2013) Mutations in prion-like domains in hnRNPA2B1 and hnRNPA1 cause multisystem proteinopathy and ALS. Nature 495:467-473. https://doi.org/10. 1038/nature11922

39. Johnson JO, Pioro EP, Boehringer A, Chia R, Feit H, Renton AE et al (2014) Mutations in the Matrin 3 gene cause familial amyotrophic lateral sclerosis. Nat Neurosci 17:664-666. https://doi.org/10.1038/nn.3688

40. Twelvetrees AE, Pernigo S, Sanger A, Guedes-Dias P, Schiavo G, Steiner RA et al (2016) The dynamic localization of cytoplasmic dynein in neurons is driven by kinesin-1. Neuron 90:1000-1015. https://doi.org/10.1016/.neuron. 2016.04.046

41. Deng J, Wang P, Chen X, Cheng H, Liu J, Fushimi K et al (2018) FUS interacts with ATP synthase beta subunit and induces mitochondrial unfolded protein response in cellular and animal models. Proc Natl Acad Sci U S A 115:E9678-E9686. https://doi.org/10.1073/pnas.1806655115

42. Katsyuba E, Auwerx J (2017) Modulating NAD(+) metabolism, from bench to bedside. EMBO J 36:2670-2683. https://doi.org/10.15252/embj.201797135

43. Rotem N, Magen I, lonescu A, Gershoni-Emek N, Altman T, Costa CJ et al (2017) ALS along the axons - expression of coding and noncoding RNA differs in axons of ALS models. Sci Rep 7:44500. https://doi.org/10.1038/ srep44500

44. Nijssen J, Aguila J, Hoogstraaten R, Kee N, Hedlund E (2018) Axon-Seq decodes the motor axon transcriptome and its modulation in response to ALS. Stem Cell Rep 11:1565-1578. https://doi.org/10.1016/j.stemcr.2018.11. 005

45. McGoldrick P, Joyce PI, Fisher EM, Greensmith $L$ (2013) Rodent models of amyotrophic lateral sclerosis. Biochim Biophys Acta 1832:1421-1436. https:// doi.org/10.1016/j.bbadis.2013.03.012

46. Lee EB, Lee VM, Trojanowski JQ (2011) Gains or losses: molecular mechanisms of TDP43-mediated neurodegeneration. Nat Rev Neurosci 13: 38-50. https://doi.org/10.1038/nrn3121

47. Fischer LR, Culver DG, Tennant P, Davis AA, Wang M, Castellano-Sanchez A et al (2004) Amyotrophic lateral sclerosis is a distal axonopathy: evidence in mice and man. Exp Neurol 185:232-240. https://doi.org/10.1016/j.expneurol. 2003.10.004

48. Ebstein SY, Yagudayeva I, Shneider NA (2019) Mutant TDP-43 causes earlystage dose-dependent motor neuron degeneration in a TARDBP Knockin mouse model of ALS. Cell Rep 26:364-373. https://doi.org/10.1016/j.celrep. 2018.12.045

49. Morrison RS, Kinoshita Y, Johnson MD, Ghatan S, Ho JT, Garden G (2002) Neuronal survival and cell death signaling pathways. Adv Exp Med Biol 513: 41-86. https://doi.org/10.1007/978-1-4615-0123-7_2

50. Kim S, Koh H (2017) Role of FOXO transcription factors in crosstalk between mitochondria and the nucleus. J Bioenerg Biomembr 49:335-341. https:// doi.org/10.1007/s10863-017-9705-0

51. Papadia S, Soriano FX, Leveille F, Martel MA, Dakin KA, Hansen HH et al (2008) Synaptic NMDA receptor activity boosts intrinsic antioxidant defenses. Nat Neurosci 11:476-487. https://doi.org/10.1038/nn2071

52. de Candia P, Blekhman R, Chabot AE, Oshlack A, Gilad Y (2008) A combination of genomic approaches reveals the role of FOXO1a in regulating an oxidative stress response pathway. PLoS One 3:e1670. https:// doi.org/10.1371/journal.pone.0001670

53. Kibbe C, Chen J, Xu G, Jing G, Shalev A (2013) FOXO1 competes with carbohydrate response element-binding protein (ChREBP) and inhibits thioredoxin-interacting protein (TXNIP) transcription in pancreatic beta cells. J Biol Chem 288:23194-23202. https://doi.org/10.1074/jbc.M1 13.473082

54. Zhang T, Baldie G, Periz G, Wang J (2014) RNA-processing protein TDP-43 regulates FOXO-dependent protein quality control in stress response. PLoS Genet 10:e1004693. https://doi.org/10.1371/journal.pgen.1004693

55. Kwak JY, Ham HJ, Kim CM, Hwang ES (2015) Nicotinamide exerts antioxidative effects on senescent cells. Mol Cells 38:229-235. https://doi. org/10.14348/molcells.2015.2253

56. Gautam M, Jara JH, Kocak N, Rylaarsdam LE, Kim KD, Bigio EH et al (2019) Mitochondria, ER, and nuclear membrane defects reveal early mechanisms for upper motor neuron vulnerability with respect to TDP-43 pathology. Acta Neuropathol 137:47-69. https://doi.org/10.1007/s00401-018-1934-8

57. Izumikawa K, Nobe Y, Yoshikawa H, Ishikawa H, Miura Y, Nakayama $H$ et al (2017) TDP-43 stabilises the processing intermediates of mitochondria transcripts. Sci Rep 7:7709. https://doi.org/10.1038/s41598-017-06953-y
58. Onesto E, Colombrita C, Gumina V, Borghi MO, Dusi S, Doretti A et al (2016) Gene-specific mitochondria dysfunctions in human TARDBP and C9ORF72 fibroblasts. Acta Neuropathol Commun 4:47. https://doi.org/10.1186/s40478016-0316-5

59. Tank EM, Figueroa-Romero C, Hinder LM, Bedi K, Archbold HC, Li X et al (2018) Abnormal RNA stability in amyotrophic lateral sclerosis. Nat Commun 9:2845. https://doi.org/10.1038/s41467-018-05049-z

60. Wang W, Arakawa H, Wang L, Okolo O, Siedlak SL, Jiang Y et al (2017) Motor-coordinative and cognitive dysfunction caused by mutant TDP-43 could be reversed by inhibiting its mitochondrial localization. Mol Ther 25: 127-139. https://doi.org/10.1016/.ymthe.2016.10.013

61. Kreiter N, Pal A, Lojewski X, Corcia P, Naujock M, Reinhardt P et al (2018) Age-dependent neurodegeneration and organelle transport deficiencies in mutant TDP43 patient-derived neurons are independent of TDP43 aggregation. Neurobiol Dis 115:167-181. https://doi.org/10.1016/..nbd.2018. 03.010

62. Magrané J, Cortez C, Gan WB, Manfredi G (2014) Abnormal mitochondrial transport and morphology are common pathological denominators in SOD1 and TDP43 ALS mouse models. Hum Mol Genet 23:1413-1424. https://doi.org/10.1093/hmg/ddt528

63. Haigis MC, Sinclair DA (2010) Mammalian sirtuins: biological insights and disease relevance. Annu Rev Pathol 5:253-295. https://doi.org/10.1146/ annurev.pathol.4.110807.092250

64. Outeiro TF, Kontopoulos E, Altmann SM, Kufareva I, Strathearn KE, Amore AM et al (2007) Sirtuin 2 inhibitors rescue alpha-synuclein-mediated toxicity in models of Parkinson's disease. Science 317:516-519. https://doi.org/10. 1126/science.1143780

65. Donmez G, Outeiro TF (2013) SIRT1 and SIRT2: emerging targets in neurodegeneration. EMBO Mol Med 5:344-352. https://doi.org/10.1002/ emmm.201302451

66. Blacher E, Bashiardes S, Shapiro H, Rothschild D, Mor U, Dori-Bachash M et al (2019) Potential roles of gut microbiome and metabolites in modulating ALS in mice. Nature 572:474-480. https://doi.org/10.1038/ s41586-019-1443-5

67. Fiesel FC, Schurr C, Weber SS, Kahle PJ (2011) TDP-43 knockdown impairs neurite outgrowth dependent on its target histone deacetylase 6. Mol Neurodegener 6:64. https://doi.org/10.1186/1750-1326-6-64

68. Klim JR, Williams LA, Limone F, San Juan IG, Davis-Dusenbery BN, Mordes DA et al (2019) ALS-implicated protein TDP-43 sustains levels of STMN2, a mediator of motor neuron growth and repair. Nat Neurosci 22:167-179. https://doi.org/10.1038/s41593-018-0300-4

69. Tripathi VB, Baskaran P, Shaw CE, Guthrie S (2014) Tar DNA-binding protein43 (TDP-43) regulates axon growth in vitro and in vivo. Neurobiol Dis 65: 25-34. https://doi.org/10.1016/j.nbd.2014.01.004

70. Jablonka S, Beck M, Lechner BD, Mayer C, Sendtner M (2007) Defective $\mathrm{Ca} 2+$ channel clustering in axon terminals disturbs excitability in motoneurons in spinal muscular atrophy. J Cell Biol 179:139-149. https:// doi.org/10.1083/jcb.200703187

71. Moradi M, Sivadasan R, Saal L, Luningschror P, Dombert B, Rathod RJ et al (2017) Differential roles of alpha-, beta-, and gamma-actin in axon growth and collateral branch formation in motoneurons. J Cell Biol 216:793-814. https://doi.org/10.1083/jcb.201604117

72. Rathod R, Havlicek S, Frank N, Blum R, Sendtner M (2012) Laminin induced local axonal translation of beta-actin mRNA is impaired in SMN-deficient motoneurons. Histochem Cell Biol 138:737-748. https://doi.org/10.1007/ s00418-012-0989-1

73. Rossoll W, Jablonka S, Andreassi C, Kroning AK, Karle K, Monani UR et al (2003) Smn, the spinal muscular atrophy-determining gene product, modulates axon growth and localization of beta-actin mRNA in growth cones of motoneurons. J Cell Biol 163:801-812. https://doi.org/10.1083/jcb. 200304128

74. Sivadasan R, Hornburg D, Drepper C, Frank N, Jablonka S, Hansel A et al (2016) C9ORF72 interaction with cofilin modulates actin dynamics in motor neurons. Nat Neurosci 19:1610-1618. https://doi.org/10.1038/nn.4407

\section{Publisher's Note}

Springer Nature remains neutral with regard to jurisdictional claims in published maps and institutional affiliations. 